\title{
AIDS-associated Kaposi sarcoma in Uganda: response to treatment with highly active antiretroviral therapy and chemotherapy HQ Nguyen ${ }^{1}$, F Okuku ${ }^{6}$, F Ssewankambo ${ }^{7}$, AS Magaret ${ }^{2,3,4}$, C Johnston 1,5, A Wald ${ }^{1,2,3}$, A Kambugu 7 , L Corey $2,3,5$, J Orem ${ }^{6}$ and C Casper*1,3,5
}

\begin{abstract}
Address: ${ }^{1}$ Department of Epidemiology, University of Washington; Seattle, Washington, USA, ${ }^{2}$ Department of Laboratory Medicine, University of Washington; Seattle, Washington, USA, ${ }^{3}$ Deparment of Medicine, University of Washington; Seattle, Washington, USA, ${ }^{4}$ Program in Biostatistics, Fred Hutchinson Cancer Research Center, Seattle, Washington, USA, 5 Program in Vaccine and Infectious Disease Institute, Fred Hutchinson Cancer Research Center, Seattle, Washington; USA, ${ }^{6}$ Uganda Cancer Institute, Kampala, Uganda and 7 Infectious Disease Institute, Kampala, Uganda

* Corresponding author
\end{abstract}

from I I th International Conference on Malignancies in AIDS and Other Acquired Immunodeficiencies (ICMAOI): Basic, Epidemiologic, and Clinical Research

Bethesda, MD, USA. 6-7 October 2008

Published: 17 June 2009

Infectious Agents and Cancer 2009, 4(Suppl 2):O5 doi:10.II86/I750-9378-4-S2-O5

This abstract is available from: http://www.infectagentscancer.com/content/4/S2/O5

(C) 2009 Nguyen et al; licensee BioMed Central Ltd.

\section{Background}

Highly active antiretroviral therapy (HAART) alone or in combination with systemic chemotherapy has been shown to be effective therapy for AIDS-associated Kaposi sarcoma (KS) in resource-rich countries. It is not known whether HAART or chemotherapy regimens available in resource-poor regions are effective in areas where KS is hyperendemic and typically more aggressive. Therefore, we conducted a retrospective cohort study to evaluate clinical response among patients with AIDS-associated KS treated with HAART alone or in combination with chemotherapy in Uganda.

\section{Methods}

Records of patients with AIDS-associated KS who had attended the Infectious Disease Institute (IDI) in Kampala, Uganda, for HIV care between January 2004 and December 2006 were linked to records at the Uganda Cancer Institute (UCI). Patients $\geq 18$ years were eligible if they had histologically or clinically confirmed KS, HIV infection, and $\geq 1 \mathrm{KS}$ visit after the initial diagnosis prior to December 2006. Demographic and clinical information were abstracted from records at both institutions. CD4 T-cell count during follow-up and HAART information was obtained from the IDI, while chemotherapy details were obtained from the UCI. Cox's proportional hazards models were used to identify predictors of improvement and resolution of KS disease separately.

\section{Results}

We identified 177 patients with a diagnosis of KS and $\geq 1$ KS visit after diagnosis at either the IDI or UCI. Approximately half (53\%) were female and the median age was 35 years. At the time of KS diagnosis, 94 (50\%) had a BMI $<18.5 \mathrm{~kg} / \mathrm{m}^{2}, 150(85 \%)$ had a Karnofsky score $\geq 70,95$ (54\%) had T1 tumor stage, 45 (25\%) had macular lesions, $50(28 \%)$ had nodular lesions, and $10(6 \%)$ had fungating lesions. Ninety-six (54\%) had KS lesions in $>1$ location and $66(37 \%)$ had $\geq 10$ lesions. The median CD4 Tcell count was 87 cells $/ \mathrm{mm}^{3}$ (Interquartile range (IQR): 17-230). One year following KS diagnosis, the cumulative probability of improvement was 0.71 (95\% CI: $0.63-$ 0.79 ) and resolution was 0.08 (95\% CI: 0.03-0.13). After two years, the cumulative probability of resolution was 0.28 (95\% CI: 0.17-0.39). In univariate analyses, none of the baseline characteristics (sex, age, employment status, body mass index (BMI), Karnofsky score, and CD4 T-cell count) were associated with either improvement or resolution. Three clinical factors were associated with improvement: having lesions on the upper extremities $(\mathrm{HR}=1.7,95 \% \mathrm{CI}: 1.1-2.6, \mathrm{p}=0.001)$, lesions on the hard palate $(\mathrm{HR}=1.5,95 \% \mathrm{CI}: 1.0-2.3, \mathrm{p}=0.05)$, and lesions in $>1$ location $(\mathrm{HR}=1.6,95 \% \mathrm{CI}$ : $1.0-2.5, \mathrm{p}=$ 
0.05). Three different clinical factors were associated with a decreased risk of resolution in univariate analyses: having nodular lesions $(\mathrm{HR}=0.19,95 \% \mathrm{CI}$ : 0.04-0.87, $\mathrm{p}=$ 0.03 ), lesions on the lower extremities ( $\mathrm{HR}=0.38,95 \%$ CI: $0.15-0.96, p=0.04)$, and $\geq 10$ lesions ( $\mathrm{HR}=0.30,95 \%$ CI: 0.09-0.94, p =0.04). Both HAART and chemotherapy were associated with improvement of disease, but only HAART was associated with resolution in univariate analyses. In multivariate analyses, male sex was the only variable other than HAART and chemotherapy that was independently associated with increased risk of improvement $(\mathrm{HR}=2.0,95 \% \mathrm{CI}: 1.3-3.1, \mathrm{p}=0.002)$. For resolution, having a low BMI $(<18.5, \mathrm{HR}=0.15,95 \% \mathrm{CI}$ : $0.06-$ $0.39, \mathrm{p}<0.0001)$ and lesions located on the lower extremities ( $\mathrm{HR}=0.12,95 \% \mathrm{CI}: 0.03-0.43, \mathrm{p}=0.001$ ) were independently associated with a decreased risk of disease resolution. Among patients on HAART, those receiving efavirenz- and protease inhibitor-containing HAART regimens were 6.9 (95\% CI: $1.8-27, \mathrm{p}=0.006)$ and 14 times (95\% CI: $1.2-172, \mathrm{p}=0.04)$ more likely to experience disease resolution compared to those receiving Triomune (stavudine, lamivudine, nevirapine). There was a trend towards a better chance of KS resolution with increasing dosage of chemotherapy $(\mathrm{HR}=1.02,95 \% \mathrm{CI}: 1.00-1.05$, $\mathrm{p}=0.1)$.

\section{Conclusion}

HAART, used alone or in combination with systemic chemotherapy, is effective therapy for epidemic KS in Uganda. While the majority of patients experience improvements in KS lesions during the first two years of therapy, a very small minority resolved their disease. Factors such as the burden and location of lesions, gender, and under-nutrition may impact the success of KS therapy. Our data also suggest that the individual components of HAART regimens may have differential effects on KS response, with the most widely available drug in resourcelimited settings (Triomune) showing less effectiveness than other antiretroviral combinations. Additional studies are required to define the optimal treatment of KS in endemic areas.

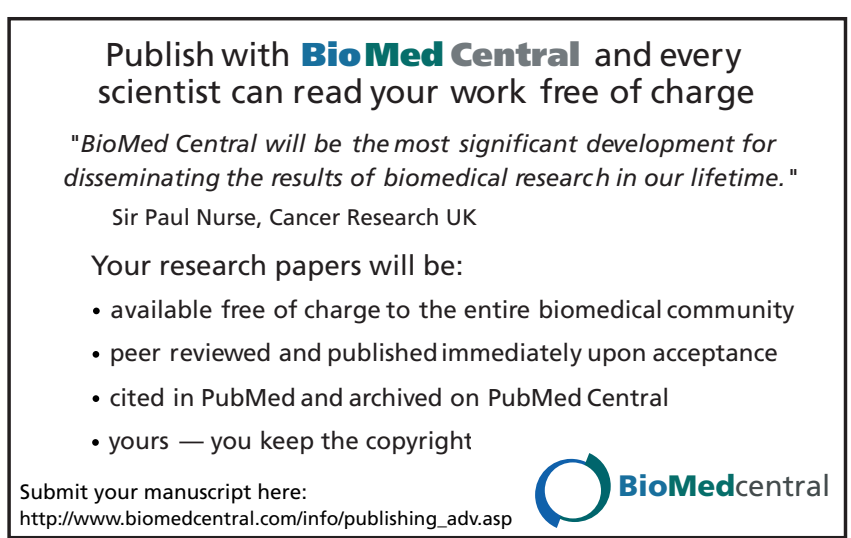

Page 2 of 2

(page number not for citation purposes) 